\title{
Annual Acknowledgement to Reviewers
}

\section{Sigmund Hough}

(C) Springer Science+Business Media, LLC 2012

The journal of Sexuality and Disability wishes to thank the Editorial Board Members and Selected Peer Reviewers for their expertise and commitment during the year 2011. 